\title{
Organophotocatalytic Synthesis of Phosphoramidates
}

\author{
Marta Meazza, ${ }^{\mathrm{a}}$ Agnieszka Kowalczuk, ${ }^{\mathrm{a}}$ Luke Shirley, ${ }^{\mathrm{a}}$ Jung Woon Yang, ${ }^{\mathrm{a}, \mathrm{b}}$ Hao Guo, ${ }^{\mathrm{c}, *}$ \\ and Ramon Rios ${ }^{\mathrm{a}, \mathrm{b}, *}$
}

a Faculty of Natural and Environmental Science, School of Chemistry, University of Southampton, Highfield Campus, Southampton SO17 1BJ, United Kingdom

E-mail: R.Rios-Torres@ southampton.ac.uk

b Department of Energy Science, Sungkyunkwan University, Suwon 440-746, Korea

c Department of Chemistry, Fudan University, 220 Handan Road, Shanghai, 200433, Fudan, China

Received: ((will be filled in by the editorial staff))

Supporting information for this article is available on the WWW under http://dx.doi.org/10.1002/adsc.201\#\#\#\#\#\#.((Please delete if not appropriate))

\begin{abstract}
We describe the use of visible light in conjunction with an organic dye for the synthesis of phosphoramidates. Cross dehydrogenative coupling reactions between phosphites and amines are reported using organic dyes and light as catalysts for the first time. It is not only a novel application of organic dyes but also fulfils the requirement of sustainability and green chemistry avoiding the use of chromatographic purification technique. The product was simply isolated from the reaction mixture via acid-base workup procedure, rendering the pure product in good yields.
\end{abstract}

Keywords: phosphoramidates; CDC; Green Chemistry; photocatalysis; dye

The quest for green procedures has become of paramount importance for organic chemists in the last decade. Discovery benign, green and atom economic processes is one of the corner stones for chemists nowadays. ${ }^{[1]}$ One of the most promising eco-friendly processes is the use of light in combination with a photosensitizer to catalyze organic reactions. Advantages to use light as a source of energy are that it is a renewable source, clean, non-toxic and cheap. For these reasons, the use of photocatalysis has grown exponentially in recent years. ${ }^{[2]}$

For similar reasons, cross dehydrogenative couplings (CDCs) have become a hot topic of interest for chemists. ${ }^{[3]}$ CDCs present some advantages such as no requirement of prefunctionalized starting materials and typically highly efficient, atomeconomic processes, which should be an excellent way to shorten the common synthetic routes or to introduce late-functionalization for interesting scaffolds. In our research group, we are interested in the development of green organocatalytic reactions that allow us to build new atom-atom $\sigma$-bonds. To do this, photocatalytic reactions for CDC have attracted our attention. Inspired by previous works of Rueping $^{[4]}$ among others, we envisioned that photocatalysis, using organic dyes, could be contributing to the development of new reactions for the synthesis of phosphoramidates.

Phosphoramidates are very important structural scaffolds in many biologically active molecules ${ }^{[5]}$ and industrially important products. ${ }^{[6]}$ Moreover, in recent years, phosphoramidates have become important chiral ligands for several metal-catalyzed reactions. ${ }^{[7]}$ In medicinal chemistry, phosphoramidate ProTide technology has been used to bypass the rate-limiting step of the initial phosphorylation of nucleosides and to act as a better inhibitor. ${ }^{[8]}$ Recently, Zhou and coworkers have used chiral phosphoramidates as organocatalysts for several reactions such as addition of oxindoles to nitrostyrenes ${ }^{[9]}$ or the Michael addition of fluorinated silyl enol ethers ${ }^{[10]}$ with excellent results, showing the importance of this type of functional group as catalyst scaffold.

Despite the importance of phosphoramidates, their synthesis requires harsh conditions and/or the use of halides or precious transition metals. Typical methodologies for the synthesis of phosphoramidates are described as follows: (i) they were conventionally synthesized by the reaction of amine with the appropriate phosphorous halide. ${ }^{[1]}$ Generally, these kinds of phosphorous reagents are moisture sensitive and thermally unstable; (ii) Other methods require the use of potentially explosive and toxic reagents or need multi-step synthesis. For example, the Staudingerphosphite reaction uses azides or phosphoryl azides. ${ }^{[12]}$ In addition; highly toxic chlorinating agents were used as solvents, for example, $\mathrm{CCl}_{4}$ in the Atherton-Todd reaction, to generate the reactive phosphorous halide species. In order to overcome the above-mentioned limitations, some examples of direct catalytic approaches were recently reported based on the concept of CDCs process: (i) copper-catalyzed aerobic oxidative coupling between amines and phosphonates with excellent results were developed by the groups of Hayes and Mizuno independently in 2013 [Scheme 1, (Eq. 1)]; ${ }^{[13]}$ (ii) Prabhu and coworkers reported the use of $I_{2}$ as catalyst with a 
(over)stoichiometric amounts of $\mathrm{H}_{2} \mathrm{O}_{2}$ as oxidant to obtain phosphoramidates in good yields [Scheme 1, (Eq. 2)]. ${ }^{[14]}$ From a practical and green chemistry point of view, however, a general solutions such as the elimination of the use of precious transition metals or stoichiometric oxidants are still highly desirable.

Herein, we report a simple, non-toxic, and transition-metal-free, a P-N bond-forming CDC reactions between phosphites and amines for the synthesis of phosphoramidates in a one-pot manner using an organic dye as photocatalyst and air as oxidant under irradiation of green light-emitting diode (LED) [Scheme 1, (Eq. 3)]. Moreover, large scale production of the desired product is demonstrated without the need of chromatographic purification.

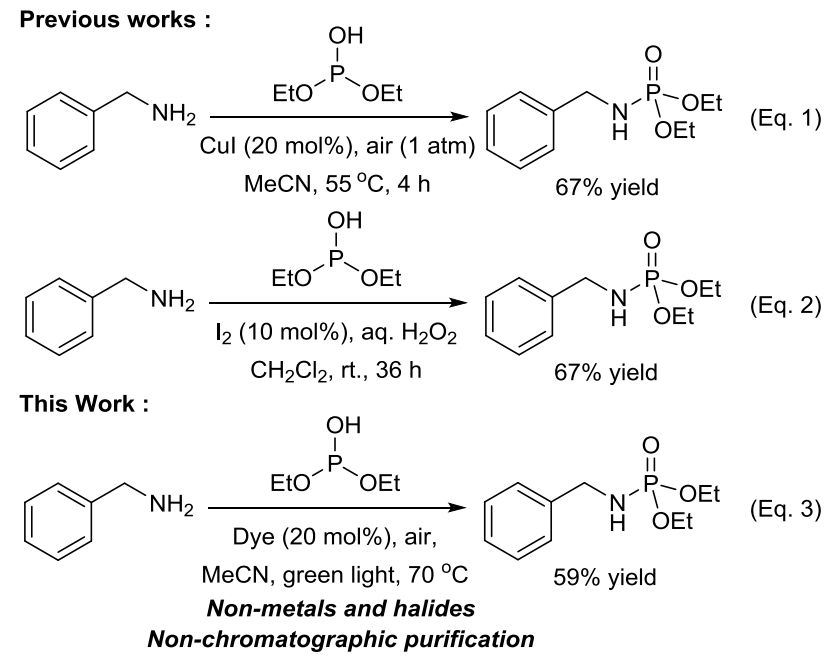

Scheme 1. Previous works and our work.

We initially commenced CDC reaction between diethylphosphite 1a and $p$-anisidine $\mathbf{2 a}$ in acetonitrile under aerobic conditions, catalyzing by several organic dyes at room temperature (Table 1). The reaction furnished phosphoramidate 3a with low conversion at room temperature. Among organic dyes, Rose Bengal (III) was proven to be the best dye in combination with green light for this study whereas other dyes such as Eosyn Y (I) or Methylene Blue (II), surprisingly, did not render any product (Table 1, entries 1-2). After screening of several solvents, it revealed that toluene gave comparable result to that obtained in acetonitrile at room temperature (Table 1, entries 3-4). On the other hand, the use of chloroform, EtOAc, and DMSO solvents gave lower yields (Table 1 , entries 5-7). In order to improve the reaction rate and the yields, the reaction was carried out in either toluene or acetonitrile at $70{ }^{\circ} \mathrm{C}$. Acetonitrile gave striking difference in terms of the yield compared with toluene, giving the desired product in almost quantitative yield (95\%) (Table 1, entry 9). Control reactions demonstrated that no reaction occurred in the absence of organic dye III or light source (Table 1, entries 10 and 11).
Having the optimal conditions in hand, we focused our attention on the reaction scope of a variety of aromatic and aliphatic amines with 1a.

Table 1. Screening of parameters. ${ }^{[\mathrm{a}]}$

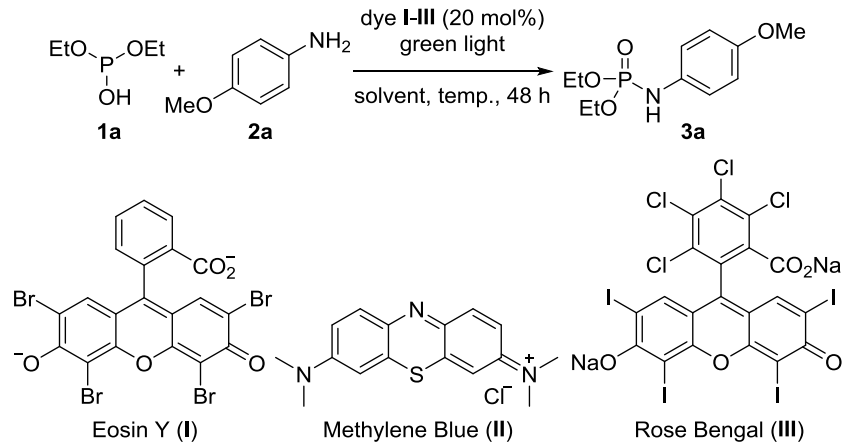

\begin{tabular}{llllll}
\hline Entry & Dye & $\begin{array}{l}\text { Green } \\
\text { light }\end{array}$ & Solvent & $\begin{array}{l}\text { Temp. } \\
{\left[{ }^{\circ} \mathrm{C}\right]}\end{array}$ & $\begin{array}{l}\text { Yield } \\
{[\%]^{\text {b] }]}}\end{array}$ \\
\hline 1 & I & yes & $\mathrm{CH}_{3} \mathrm{CN}$ & $\mathrm{RT}$ & $\mathrm{NR}$ \\
2 & II & yes & $\mathrm{CH}_{3} \mathrm{CN}$ & $\mathrm{RT}$ & $\mathrm{NR}$ \\
3 & III & yes & $\mathrm{CH}_{3} \mathrm{CN}$ & $\mathrm{RT}$ & 36 \\
4 & III & yes & Toluene & $\mathrm{RT}$ & 40 \\
5 & III & yes & $\mathrm{CHCl}_{3}$ & $\mathrm{RT}$ & 26 \\
6 & III & yes & EtOAc & $\mathrm{RT}$ & 13 \\
7 & III & yes & DMSO & $\mathrm{RT}$ & 17 \\
8 & III & yes & Toluene & 70 & 54 \\
9 & III & yes & $\mathrm{CH}_{3} \mathrm{CN}$ & 70 & 95 \\
10 & - & yes & $\mathrm{CH}_{3} \mathrm{CN}$ & $\mathrm{RT}$ & $\mathrm{NR}$ \\
11 & III & no & $\mathrm{CH}_{3} \mathrm{CN}$ & $\mathrm{RT}$ & $\mathrm{NR}$ \\
\hline \multicolumn{5}{l|}{ Reaction conditions: $\mathbf{1 a}(0.362 \mathrm{mmol}), \mathbf{2 a}(0.724 \mathrm{mmol})$, }
\end{tabular}
dye I-III (20 mol\%), air (1 atm), green light, solvent (1.5 $\mathrm{mL}) .{ }^{[\mathrm{b}]}$ Isolated yields.

As shown in Scheme 2, the use of electrondonating substituents, such as 4-OMe (3a), 3,4,5$\mathrm{OMe}(\mathbf{3 m})$, on the aromatic ring of the anilines produced the corresponding phosphoramidates in high yields, whilst the use of 2-OMe substituent gave slightly lower yields. When methyl substituted anilines were employed, the final products were obtained in almost quantitative yields ( $p$-methyl aniline-derived product 3c, 97\%; $m$-methyl anilinederived product 3d, 99\%; o-methyl aniline-derived product 3e, 98\%) regardless of the substituent's position. When an electron-withdrawing substituted aniline (3k) and sterically congested 1-naphthylamine (3l) were used, moderate yields were achieved. We also examined the functional group tolerance with halogenated aromatics $(\mathbf{3 f}-\mathbf{j})$. All of the halogenated anilines produced the desired products with extremely high yields under our reaction conditions: 4-F (3f) 73\%; 4-Cl (3g) 74\%; 4-Br (3h) 87\%; 4-I (3i) 96\%; 2$\mathrm{F}(\mathbf{3 j}) 93 \%$.

The only limitation of the present methodology is the use of terminal vinyl aniline because of its sensitive radical nature which may give oligo- or polymerization by radical cations. It is noteworthy that the pure products were obtained in most cases 
after simple acid-base workup procedure without any need for additional purification technique.

$$
\text { 2a-o }
$$
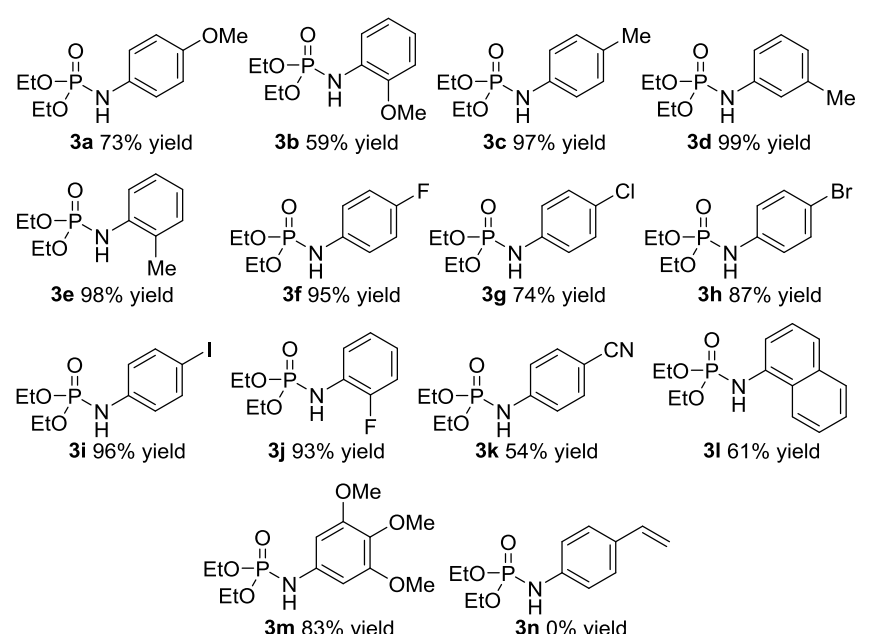

Scheme 2. Substrate scope of aromatic amines.

Next, we investigated the reaction scope with more challenging aliphatic amine substrates. Previously, aliphatic amine has been introduced in a similar CDC reaction with diethylphosphite under organic dyecatalyzed photoredox reactions by the group of Rueping. However, the Kabachnik-Fields (K-F) product (i.e. $\alpha$-amino phosphonate) was observed via $\mathrm{C}-\mathrm{P}$ bond formation during the course of the reaction [Scheme 3, (Eq. 1)].
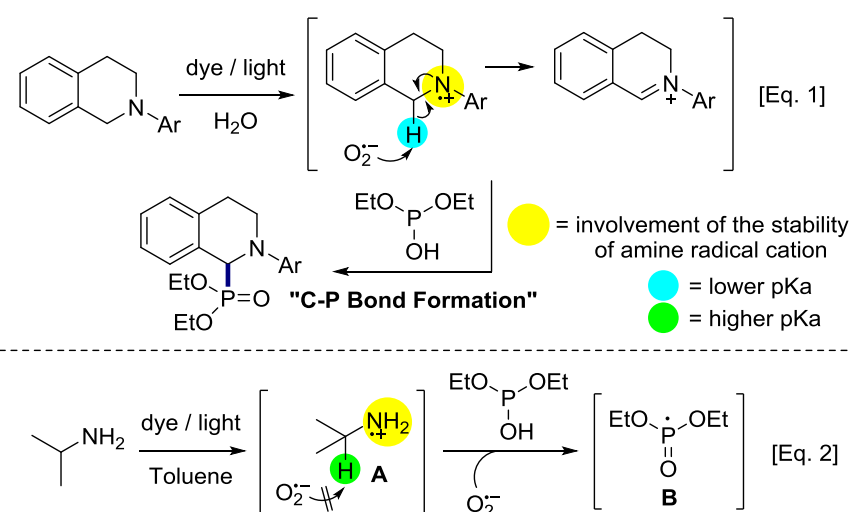

$$
A+B \longrightarrow \text { EtO- }{ }_{\text {EtO' }}^{\text {II }}
$$

"N-P Bond Formation"

Scheme 3. Competitive reactions between C-P and N-P bond formation.
In contrast to the work of Rueping, phosphoramidate products (3o-q) were selectively rendered in reasonable yields (42-59\%) via N-P bond formation when the reaction was performed in toluene under our optimized reaction conditions [Scheme 3, (Eq. 2) and Scheme 4]. These results are dependent on the stabilization of tertiary or primary amine radical cations, followed by the subsequent deprotonation efficiency of proton at the $\alpha$-position of amine radical cations by base (e.g. superoxide anion), which is governed by $\mathrm{pKa}$ value of the relevant acidic protons. For example, when tetrahydroisoquinoline was used, a mixture of the K-F product and the phosphoramidate was found (14:1 ratio).

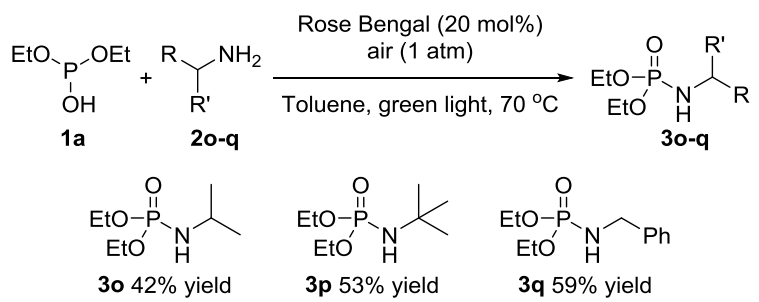

Scheme 4. Substrate scope of aliphatic amines.

A proposed mechanism for the reaction is outlined in Scheme 5. The organic dye (Rose Bengal, III) accepts a photon from the visible-light source to populate the excited-state dye (IV), which removes one electron from the nitrogen atom of $\mathbf{2}$ to generate amine radical cation 4 by a single-electron transfer (SET) process. The radical anion dye $\mathbf{V}$ is re-oxidized to the groundstate dye by molecular oxygen, forming the superoxide anion 5. Next, the superoxide anion $\mathbf{5}$ reacts with the phosphite 1a to liberate the phosphoryl radical 7 and hydrogen peroxide anion $\mathbf{6}$. The final target molecule $\mathbf{3}$ is produced by the reaction of phosphoryl radical $\mathbf{7}$ and amine radical cation $\mathbf{4}$, followed by the deprotonation of the protonated phosphoramidate 8 by hydrogen peroxide anion 6 .

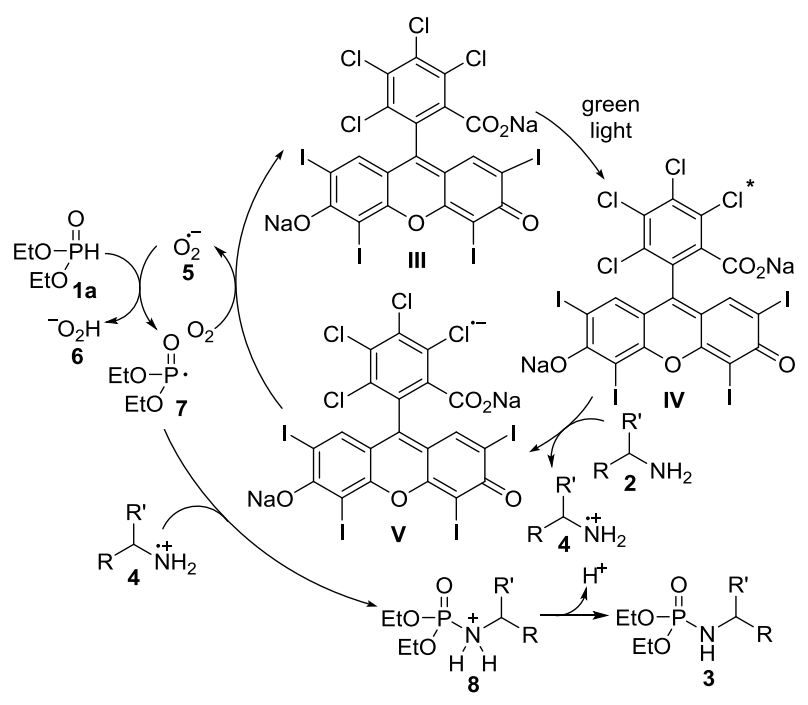


Scheme 5. Plausible mechanism for the synthesis of phosphoramidate.

To verify the stabilization of amine radical cation species, we decided to perform the reaction with equal molar ratio of $p$-anisidine and $p$-cyanoaniline in a single flask under the optimal reaction conditions. Gratifyingly, the phosphoramidate 3a derived from $p$ anisidine was exclusively formed, indicating that a better ability of electron-donating group such as OMe to stabilize amine radical cation preferentially (Scheme 6). In order to prove the radical nature of the intermediates, the reaction was conducted with 1 equiv. of TEMPO. In this conditions no product was obtained, showing clearly that an intermediate radical is formed. Another fact that validate our mechanistic proposal of the formation of intermediate $\mathbf{4}$ is the mixture of phosphoramidation and K-F products when tetrahydroisoquinoline was used.

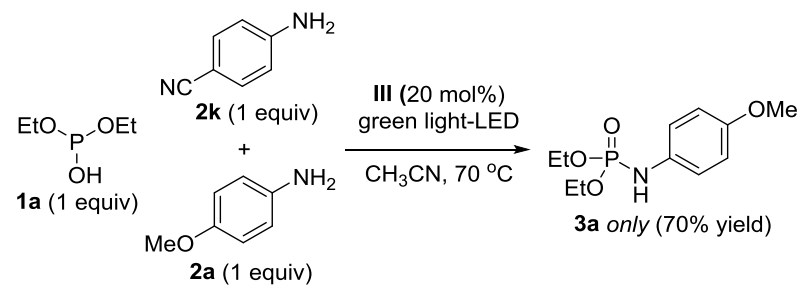

Scheme 6. Selective formation of phosphoramidate product 3a via the stabilization of amine radical cation species by controlling electronic properties of substituents on the aromatic ring of anilines.

The recyclability of Rose Bengal was also examined. Organic dye catalyst was recovered and reused after completion of the reaction by simple acid-base extraction. The recovered catalyst exhibited similar results to those obtained in the first cycle; first cycle (74\% yield) and second cycle (67\% yield).

Finally, we applied this protocol to the large-scale production of the phosphoramidate $\mathbf{3 i}$ derived from $p$ iodoaniline (Scheme 7). The reaction was repeated on a $5.00 \mathrm{~g}$ scale of diethylphosphite $1 \mathrm{a}$ with 2 equiv. of $p$-iodoaniline $2 \mathbf{i}$. The product $3 \mathbf{i}$ was isolated in $82 \%$ yield without any assistant of chromatographic purification.

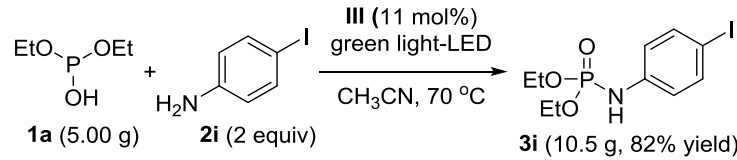

Scheme 7. Large-scale production of $\mathbf{3 i}$.

In summary, we have developed a new methodology for the synthesis of phosphoramidates catalyzed by organic dyes and light (a renewable energy) with excellent results. This methodology complements and improves the existing technologies eliminating the use of metals or halide reagents and, importantly, the requirement of tedious purification steps. For these reasons, we believe that this new process fulfills all the requirements of green chemistry and opens a new gate to the development of more sustainable methodologies based on these observations. Further studies towards the use of more reactive dyes and expanding the scope of the reaction to other interesting compounds (alcohols, amides, thiols, etc.) are ongoing in our laboratory.

\section{Experimental Section}

In a closed vial were added in this sequence: the organic dye Rose Bengal (73 mg, $0.072 \mathrm{mmol}, 20$ mol\%), the amine $(0.724 \mathrm{mmol}, 2$ equiv), the solvent $(1.5 \mathrm{~mL})$ and diethyl phosphate $(47 \mu \mathrm{L}, 0.362 \mathrm{mmol}$, 1 equiv). The reaction mixture was stirred at $70{ }^{\circ} \mathrm{C}$ in the photoreactor under green light (see table of results for reaction times). $\mathrm{CHCl}_{3}$ was added to the crude mixture and the organic phase was washed with $\mathrm{HCl}$ $0.5 \mathrm{M}(3 \times 20 \mathrm{~mL})$, then with saturated $\mathrm{NaHCO}_{3}$ aqueous solution $(3 \times 20 \mathrm{~mL})$. The organic phases were dried over $\mathrm{MgSO}_{4}$, filtered and the solvent was evaporated under vacuo to afford the desired phosphoramidate.

\section{Acknowledgements}

M.M. and R.R acknowledge the European Regional Development Fund (ERDF) for co-financing the AI-CHEM -Chem project (No. 4061) through the INTERREG IV A France (Channel) - England cross-border cooperation Programme. M.M., A. K., L.S. and R.R. are grateful for the EPSRC Core Capability Funding (EP/K039466/1). J.W.Y. thanks the NRF Nano.Material Technology Development Program (2012M3A7B4049652) for financial support. R.R., M.M., and L.S. thanks EU for a CIG grant. FP7-MC-CIG-618237.

\section{References}

[1] B. M. Trost, Angew. Chem. Int. Ed. 1995, 34, 259-281.

[2] For reviews on photoredox catalysis, see: a) C. K. Prier, D. A. Rankic, D. W. C. MacMillan, Chem. Rev. 2013, 113, 5322-5363; b) T. P. Yoon, M. A. Ischay, J. Du, Nat. Chem. 2010, 2, 527-532.

[3]R. Narayan, K. Matcha, A. P. Antonchick, Chem. Eur. J. 2015, 21, 14678-14693; b) C.-J. Li, Z. Li, Pure Appl. Chem. 2006, 78, 935-945.

[4] M. Rueping, S. Zhu, R. M. Koenigs, Chem. Commun. 2011, 47, 8679-8681.

[5] a) D. R. Phillips, M. Uramoto, K. Isono, J. A. McCloskey, J. Org. Chem. 1993, 58, 854-859; b) J. I. Guijarro, J. E. González-Pastor, F. Baleux, J. L. San Millán, M. A. Castilla, M. Rico, F. Moreno, M. Delepierre, J. Biol. Chem. 1995, 270, 23520-23532; c) 
R. A. Dwek, Chem. Rev. 1996, 96, 683-720; d) M. J. Berridge, R. F. Irvine, Nature 1989, 341, 197-205; e) M. J. Berridge, R. F. Irvine, Nature 1984, 312, 315-321.

[6] a) P. Garcia, Y. Y. Lau, M. R. Perry, L. L. Schafer, Angew. Chem. Int. Ed. 2013, 52, 9144-9148; b) T.-M. Nguyen, S. Chang, B. Condon, R. Slopek, E. Graves, M. Yoshioka-Tarver, Ind. Eng. Chem. Res. 2013, 52, 47154724.

[7] a) S. E. Denmark, G. L. Beutner, Angew. Chem. Int. Ed. 2008, 47, 1560-1638; b) O. Molt, T. Schrader, Synthesis 2002, 18, 2633-2670.

[8] R. F. Roush, E. M. Nolan, F. Löhr, C. T. Walsh, J. Am. Chem. Soc. 2008, 130, 3603-3609.

[9] M. Ding, F. Zhou, Y.-L. Liu, C.-H. Wang, X.-L. Zhao, J. Zhou, Chem. Sci. 2011, 2, 2035-2039.

[10] J.-S. Yu, F.-M. Liao, W.-M. Gao, K. Liao, R.-L. Zuo, J. Zhou, Angew. Chem. Int. Ed. 2015, 54, 7381-7385.

[11] F. R. Atherton, H. T. Openshaw, A. R. Todd, J. Chem. Soc. 1945, 660-663.

[12] a) R. L. Letsinger, M. E. Schott, J. Am. Chem. Soc. 1981, 103, 7394-7396; b) J. Nielsen, M. H. Caruthers, $J$. Am. Chem. Soc. 1988, 110, 6275-6276; c) I. Wilkening, G. del Signore, C. P. R. Hackenberger, Chem. Commun. 2008, 2932-2934.

[13] a) J. Fraser, L. J. Wilson, R. K. Blundell, C. J. Hayes, Chem. Commun. 2013, 49, 8919-8921; b) X. Jin, K. Yamaguchi, N. Mizuno, Org. Lett. 2013, 15, 418-421.

[14] J. Dhineshkumar, K. R. Prabhu, Org. Lett. 2013, 15, 6062-6065. 


\section{COMMUNICATION}

Organophotocatalytic Synthesis of

Phosphoramidates

Adv. Synth. Catal. Year, Volume, Page - Page

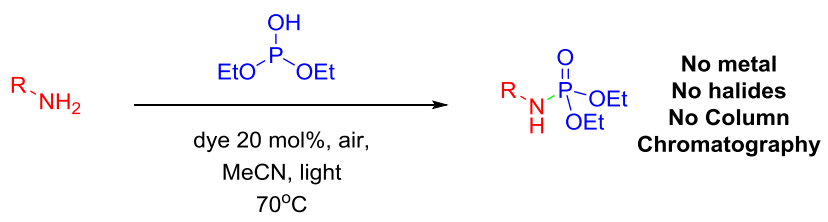

Marta Meazza, Agnieszka Kowalczuk, Luke

Shirley, Jung Woon Yang, Hao Guo,* and Ramon Rios* 